\title{
¿PUEDE EL LIDERAZGO DE PROFESORES AUMENTAR LA SATISFACCIÓN DE ESTUDIANTES? UNA REVISIÓN DE LA LITERATURA
}

\author{
Jeffrey Hanson Costa ${ }^{1 *}$; Lurdes Marlene Seide Froemming ${ }^{2}$; Fernando Oliveira de Araujo ${ }^{3}$; José Mauro \\ Gonçalves Nunes 4 \\ 1 Universidad Nacional de Misiones - UNAM, Facultad de Ciencias Económicas - FCE | Programa de Doctorado \\ en Administración, Miguel Lanús (CP N3304), Misiones, Argentina. \\ 2 Universidade Regional do Noroeste do Estado do Rio Grande do Sul - UNIJUÍ, DACEC- Departamento de \\ Ciências Administrativas, Contábeis, Econômicas e da Comunicação, Bairro Universitário, RS, Brasil. \\ 3 Universidade Federal Fluminense - UFF | Escola de Engenharia | Departamento de Engenharia de Produção | \\ Programa de Pós-Graduação em Sistemas de Gestão, São Domingos, Niterói, RJ, Brasil. \\ 4 Fundação Getulio Vargas - FGV | Escola Brasileira de Administração Pública e de Empresas| Programa de Pós- \\ Graduação em Administração, Rio de Janeiro, RJ, Brasil.
}

* jeffreycosta.br@gmail.com

\section{RESUMEN}

Esta investigación buscó analizar, recopilar y organizar las contribuciones existentes en la literatura del Liderazgo de profesores para la satisfacción de los estudiantes de las Instituciones de Educación Superior (IESs). Los hallazgos fueron organizados en un cuadro que exhibe los investigadores que produjeron evidencias de que el Liderazgo Transformacional de los docentes es capaz de aumentar la satisfacción. Además, también se encontró evidencia de que la satisfacción puede ayudar al aprendizaje de los estudiantes. Teniendo en cuenta que, en el caso de dichas instituciones, los alumnos también son clientes, estas descubiertas pueden subsidiar acciones dirigidas a aumentar su competitividad, al dejar los alumnos más satisfechos y más bien preparados (aprendizaje) para el mercado de trabajo.

\section{CAN TEACHER CLASSROOM LEADERSHIP CONTRIBUTE TO STUDENTS' SATISFACTION? A REVIEW OF THE LITERATURE}

\begin{abstract}
This research's objective was to analyze, collect and organize the existing contributions in the literature of Teachers' Leadership to the satisfaction of students of Higher Education Institutions (IESs). The findings were organized in a table showing the researchers who produced evidence that the Transformational Leadership of teachers is able to increase satisfaction. In addition, it
\end{abstract}

appears that satisfaction can help student learning, according to evidence that has also been found. Considering that, in the case of these institutions, students are also customers, these findings can subsidize actions aimed at increasing their competitiveness, with students who will then be more satisfied and even better prepared (learning) for the labor market.

KEYWORDS: Teacher Leadership; Classroom Leadership; Transformational Leadership; Satisfaction;

Competitiveness. 


\section{INTRODUCCIÓN}

La competencia es hoy en día un problema desafiante para las instituciones de educación superior en diversos países del mundo, debido al crecimiento de la oferta (ARSLANAGIĆKALAJDŽIĆ et al., 2014; PELLICIARI, 2013; POUNDER, 2005). SCHWANKE (2014) encontró que el avance de la comunicación masiva y la democratización del saber favorecieron un creciente aumento en el número de instituciones educativas en Brasil, a partir de la década de 1970. ARSLANAGIĆ-KALAJDŽIĆ et al. (2014) creen que, debido a las tecnologías de la información, que reducen las distancias, la oferta está creciendo aún más rápido, refiriéndose a la realidad de Bosnia y Herzegovina. MELCHOR CARDONA y BRAVO (2012) argumentan que las Instituciones de Educación Superior (IESs) de Colombia están compitiendo agresivamente y, por ello, es necesario encontrar estrategias para diferenciarse.

BRISCO et al. (2015) hicieron un estudio comparativo en universidades de Argentina y España y descubrieron que tanto en la percepción de los estudiantes como para los educadores, los factores más importantes para determinar la calidad de la educación superior son aquellos relacionados con los estudiantes - como el compromiso y la actitud del estudiante hacia el aprendizaje a la admisión a la universidad, la pasión del estudiante por el curso elegido, su participación activa en el proceso de aprendizaje durante su tiempo universitario, su conocimiento general al ingreso a la universidad; y el educador - el conocimiento de la materia, sus habilidades de comunicación y de motivación, la actualización de su formación pedagógica y su participación en actividades de investigación.

ARSLANAGIĆ-KALAJDŽIĆ et al. (2014) argumentan que los empleados de primera línea desempeñan un papel fundamental en la prestación de los servicios de una empresa, por su contacto directo con los clientes y, por lo tanto, en la evaluación de calidad de un cliente, utilizando un enfoque de marketing de servicios. En un contexto de educación superior, los autores separan los empleados de primera línea en dos tipos completamente diferentes: los profesores, encargados de la entrega del servicio básico, y el personal administrativo, que facilitan el proceso, y, así, afirman, basados en datos de Bosnia y Herzegovina, que ciertos profesores, a partir de sus éxitos en enseñar, investigar y practicar, se vuelven importantes activos y puntos de diferenciación para las instituciones educativas. SCHWANKE (2014) encuentra una realidad similar en Brasil, considerando "incuestionable que la calidad de la IES tiene fuerte relación con el nivel igualmente cualitativo de sus profesores".

De manera semejante, YAVUZ, YALÇIN y DIBEK (2017) afirman que la variable más importante a nivel escolar que tiene influencia sobre los logros de los estudiantes son las características del docente. Anteriormente, HILL, LOMAS y MACGREGOR (2003) llevaron a cabo grupos focales con estudiantes de educación superior en Inglaterra sobre sus percepciones relativas a experiencias de calidad en educación y también descubrieron que los factores más influyentes eran la calidad del profesor y los sistemas de apoyo estudiantil.

FROEMMING (2001) encontró, en su estudio con alumnos universitarios en Brasil, que el agrupamiento de atributos referentes a la Calidad Técnica es, para ellos, el que posee un mayor impacto en la evaluación de los Encuentros de Servicios - 29,12\% para la satisfacción y $26 \%$ para la insatisfacción -, siendo que la calidad técnica es descripta como la conjunción de los atributos relacionados con la habilidad técnica en la prestación de servicios: agilidad, capacidad de los empleados, obtención de resultados, eficacia, organización y desempeño de los profesores.

LEBLANC y NGUYEN (1997) también han apuntado, hace mucho tiempo, resultados que indicaban que los profesores (su desempeño y capacidad para inspirar confianza), junto con la reputación de la institución (su capacidad de fomentar un clima organizacional dirigido a atender las necesidades de sus clientes y la fuerza de su marca en el mercado) y las evidencias físicas (el diseño 
de las salas de clase, su iluminación, la apariencia del edificio, la limpieza en general, el grado en el que las salas de clase y de estudio son cómodas, la decoración y el ambiente) eran importantes en la percepción de calidad de los alumnos de una institución de enseño superior de Canadá.

DLAČIĆ et al. (2014) propusieron y probaron un modelo conceptual incorporando la calidad percibida del servicio, el valor percibido por el cliente y la intención de recompra en un contexto de enseñanza superior. Sus datos empíricos, recogidos entre estudiantes de graduación en Bosnia y Herzegovina y Croacia, apoyaron el modelo conceptual propuesto, mostrando que la calidad percibida del servicio y el valor percibido por el cliente tienen una influencia positiva y significativa en la intención de recompra.

Además, TSAI y LIN (2012) corroboraron lo que BEATTY y ZAHN (1990) ya habían descubierto: que las evaluaciones de los estudiantes son predictivas de su voluntad de inscribirse en cursos adicionales impartidos por el instructor y otros en el departamento; y la intención de recomendar el profesor, el curso o la disciplina a sus compañeros.

LEITHWOOD y SUN (2012) afirman que se ha prestado poca atención a la comprensión del impacto de las prácticas de liderazgo de profesores, y se puede añadir que menos aún a sus impactos en la satisfacción de los alumnos (clientes), ya que estos están limitados a los de la enseñanza superior. En Portugal, según CRUZ, PAYAN-CARREIRA, DOMINGUEZ (2017), los empleadores se han quejado de las brechas entre los perfiles de competencias de los graduados y las necesidades del mercado laboral, especialmente en las denominadas competencias personales (soft skills). En respuesta, algunas disciplinas de pensamiento crítico fueron incluidas en los currículos de la Educación Superior. Las investigaciones analizadas, entretanto, no presentaron un vínculo claro entre las intervenciones utilizadas para promover las habilidades de pensamiento crítico, las prácticas específicas utilizadas y los logros alcanzados.

POUNDER (2014), a su vez, afirma que el liderazgo transformacional en el aula es una posible forma de avanzar para las universidades y colegios bajo presión para mejorar la calidad de la enseñanza. Así, para mejorar la competitividad de las instituciones de enseñanza, puede ser útil trabajar el liderazgo de profesores y sus prácticas para aumentar la satisfacción de sus alumnos/ clientes y, también, quizá, la competitividad de ellos propios y la de las organizaciones que los contratarán.

La cuestión de investigación queda, entonces:

\section{¿Existen evidencias de que el liderazgo de profesores puede generar beneficios para la satisfacción de los alumnos?}

El objetivo de este artículo es llevar a cabo una investigación bibliográfica acerca del liderazgo de profesores en el aula que conduzca a una mayor satisfacción de los alumnos de las Instituciones de Enseñanza Superior (IESs) y, consecuentemente, puedan ser útiles a esas instituciones en sus esfuerzos para aumentar su competitividad en un mercado con cada vez más players.

Para mayor claridad, el presente estudio está organizado en 4 secciones: Introducción, Materiales y Métodos, Desarrollo, Resultados y Conclusión. El Desarrollo encontrase subdividido en "El liderazgo de profesores" y "El liderazgo de profesores y la satisfacción del cliente/alumno".

\section{MATERIALES Y MÉTODOS}

Esta investigación exploratoria, basada en una revisión bibliográfica, ejecutada entre el 16 de noviembre de 2014 y el 19 de junio de 2018, utilizó, a cada etapa, la búsqueda de expresiones 
seleccionadas en el Google Académico (www.scholar.google.com) y en el portal de periódico de CAPES, la Coordinación de Perfeccionamiento de Personal de Nivel Superior que es una fundación del Ministerio de Educación (MEC) que actúa en la expansión y consolidación del postgrado stricto sensu de Brasil (www.periodicos.capes.gov.br).

De los artículos encontrados, la mayoría estaba "abierta", o sea, disponible para download gratuito. Casi todos los demás fueron accesibles vía bibliotecas de instituciones de enseñanza superior.

Para comprender mejor el liderazgo educacional, las suyas múltiples interpretaciones, cómo se manifiesta y sus contribuciones a la satisfacción y al aprendizaje, las primeras palabras o expresiones clave utilizadas en los motores de búsqueda mencionadas fueron: "Liderazgo educativo", "Educational leadership", "Liderazgo instruccional", "Instructional leadership", "Liderazgo escolar", "School leadership".

A continuación, estos autores analizaron los más relevantes para ese estudio, y decidieron entonces investigar las expresiones: "Liderazgo de profesores", "Teacher leadership", "Professor leadership", siempre en busca de lo que conduzca a una mayor satisfacción y/o a un mayor aprendizaje de los alumnos de las Instituciones de Enseñanza Superior (IESs). Eso llevó a la identificación de 3 "olas" de Liderazgo de profesores (SILVA, GIMBERT y NOLAN, 2000) y a la búsqueda de las expresiones "Liderazgo de profesores en el aula", "Liderazgo de profesores en clase de aulas" y "Classroom leadership", en que se encontró la proposición de una $4^{\mathrm{a}}$ ola de Liderazgo de profesores (POUNDER, 2006), todas ellas investigadas y descritas a continuación.

En estos trabajos, toda referencia encontrada a aumentos en la satisfacción de los alumnos que contenían datos primarios era analizada y, cuando considerada relevante, incluida en la Tabla 2, Evidencias empíricas de aumentos en la satisfacción de los alumnos asociados con el liderazgo transformacional, a continuación. Todas las referencias encontradas relativas a aumentos en la satisfacción de alumnos que no contenían datos primarios eran buscadas y analizadas. Si estas, a su vez, contenían datos empíricos, entonces sus resultados se incluían en la Tabla 2.

Las excepciones fueron tres estudios, basados en meta-análisis (BALWANT, 2016; RICHARDSON, ABRAHAM y BOND, 2012; ROBBINS et al., 2004), incluidos en la Tabla 2 por su sistematización y consolidación, con base en métodos estadísticos, de evidencias empíricas de otros estudios. En particular, BALWANT (2016) defiende que, en comparación con los estudios individuales, un meta-análisis proporciona estimaciones más precisas de los efectos estudiados y favorece la generalización de los resultados.

La Tabla 1, a continuación, muestra los trabajos analizados en cada fase:

\begin{tabular}{|l|c|c|}
\hline \multicolumn{1}{|c|}{ Expresiones buscadas } & $\begin{array}{c}\text { Artículos con resúmenes } \\
\text { analizados }\end{array}$ & $\begin{array}{c}\text { Artículos } \\
\text { incluidos }\end{array}$ \\
\hline $\begin{array}{l}\text { "Liderazgo educativo", "Educational leadership", "Liderazgo instruccional", } \\
\text { "Instructional leadership", "Liderazgo escolar", "School leadership" }\end{array}$ & 29 \\
\hline "Liderazgo de profesores", "Teacher leadership", "Professor leadership" & 33 & 20 \\
\hline $\begin{array}{l}\text { "Liderazgo de profesores en el aula", "Liderazgo de profesores en clase de } \\
\text { aulas" y "Classroom leadership" }\end{array}$ & 35 & 32 \\
\hline Total (hay artículos utilizados en más de una fase de búsqueda) & & 32 \\
\hline
\end{tabular}

Tabla 1: Trabajos analizados en cada fase de esa investigación

Fuente: elaboración propia 


\section{DESARROLLO}

\section{EL LIDERAZGO DE PROFESORES}

POUNDER (2014) identificó un creciente reconocimiento de que las aulas universitarias son como pequeñas organizaciones sociales, donde los conceptos de organización y liderazgo son aplicables. También encontró evidencias de que lo mismo es válido en clases de educación física, en la educación media y hasta en la educación superior a distancia (online).

En acuerdo con él, HARRISON (2011), apoyándose en diversos autores, establece un paralelo entre el liderazgo instruccional y el liderazgo organizacional. Argumenta que los profesores influyen en los estudiantes, dan forma a su futuro desarrollo, centran la atención de estos en tareas específicas, los conducen a su campo o profesión de manera similar a cómo los líderes organizacionales influyen, inician, enfocan atención, establecen dirección y coordinan las actividades hacia una meta.

YORK-BARR y DUKE (2004) consideran que muchos autores escriben sobre el liderazgo de los profesores, aseguran su importancia y describen sus diversas formas, pero por lo general fallan en definirlo. Tal vez por eso, cuando se refiere a los profesores, el término liderazgo instruccional permita interpretaciones múltiples (NEUMERSKI, 2012; POUNDER, 2006; YORKBARR y DUKE, 2004; LEITHWOOD y RIEHL, 2003; HARRIS, 2003).

YORK-BARR y DUKE (2004) mencionan investigaciones que consideran diferentes expectativas en cuanto a sus papeles de liderazgo, diferentes estructuras y contextos. HARRIS (2003), por ejemplo, encuentra 3 tipos de liderazgo de profesores en su investigación:

- Liderazgo de estudiantes o de otros profesores: el profesor que actúa como facilitador, entrenador (incluso con el sentido de coach), mentor, especialista en currículo, creador de nuevos enfoques, líder/ motivador de grupos de estudio;

- Liderazgo de las tareas operativas: incluye mantener la escuela organizada y avanzar hacia sus objetivos a través de funciones como Jefe de Departamento, investigador o miembro de grupos de trabajo;

- Liderazgo a través de la toma de decisiones o colaboración: el profesor participa como miembro de equipos de mejora escolar o de comités; instigador de colaboración con entidades, como empresas, instituciones de educación superior, autoridades educativas locales $\mathrm{y}$ asociaciones de padres y profesores.

SILVA, GIMBERT y NOLAN (2000) vislumbraron tres "olas" del desarrollo del concepto de liderazgo instruccional de profesores, que se desvinculan progresivamente a la idea de liderazgo jerárquico (estas "olas" y este estudio también son referenciadas en las investigaciones de POUNDER, 2006, y YORK-BARR y DUKE, 2004):

- La primera ola limita el liderazgo de profesores dentro de la jerarquía de la organización formal y, simplemente, coloca el concepto cercano a la función docente; el énfasis está en el control, con los profesores vistos como meros ejecutores de las decisiones de los profesores líderes; en esta ola, el típico profesor líder era el jefe de departamento;

- La segunda ola pone más énfasis en la dimensión instruccional de la función docente, pero sigue relacionando el liderazgo de profesores a posiciones organizativas creadas formalmente, como jefe de equipo y desarrollador de currículos, separando el liderazgo de la función de enseñar y aun enfatizando el control; los desarrolladores curriculares y los diseñadores de instrucción crean materiales estandarizados para los otros profesores colocaren en práctica en el aula; 
- La tercera ola integra las nociones de la enseñanza y el liderazgo; identifica el liderazgo como un proceso más que un concepto de posición y reconoce que los profesores, en el proceso de llevar a cabo sus funciones, deben tener la oportunidad de expresar sus capacidades de liderazgo; basa el liderazgo de profesores en la profesionalidad y la colegialidad y es asociada a los profesores que mejoran el clima, el proceso educativo, la experiencia educativa de los estudiantes, siendo percibidos como excelentes en clase de aulas, y participan en la toma de decisiones para solucionar problemas de la escuela, ayudando a rediseñarlas.

NEUMERSKI (2012) refuerza que los autores relacionados con el liderazgo instruccional de profesores tienen, en general, una visión tradicional, que parece más alineada a la primera y segunda olas (SILVA, GIMBERT y NOLAN, 2000), centrada en los papeles formales, jerárquicos, integral o, parcialmente, fuera del aula, como consultores, gerentes de planes de estudios y sus cambios, jefes de departamento, mentores, coordinadores de desarrollo profesional, especialistas, entrenadores (coaches) etc. (LEITHWOOD et al., 2004; MARKS y PRINTY, 2003).

YORK-BARR y DUKE (2004) identificaron diversas concepciones de liderazgo instruccional de profesores en la literatura para concluir que, en común, estas destacan el uso de la experiencia de los profesores sobre la enseñanza y el aprendizaje para mejorar la cultura y la educación en las escuelas, de manera que se logre un mayor aprendizaje de los estudiantes. Tal punto de vista implica liderar a sus colegas, con un enfoque en la práctica docente, así como el trabajo a nivel organizacional para alinear el personal, los recursos fiscales y los materiales con el fin de mejorar la enseñanza y el aprendizaje. Ese trabajo de liderazgo involucra tres focos de desarrollo intencional: desarrollo individual, colaboración o desarrollo de equipo y desarrollo organizacional. Ellas han desarrollado y presentado un modelo conceptual de liderazgo de profesores para el aprendizaje de los alumnos en su artículo.

HARRIS (2003) destaca una pieza importante en la comprensión del término liderazgo instruccional de los profesores, realizando una distinción entre el liderazgo formal e informal:

- el liderazgo formal abarca responsabilidades como coordinador de disciplina, jefe de departamento o jefe de grado(s), a menudo alejándose el profesor de la sala de clases;

- el liderazgo informal, por otro lado, está constituido por funciones relacionadas con el aula, como la planificación, la comunicación de objetivos, la organización de actividades, la creación de un ambiente de trabajo agradable, la supervisión y motivación de los supervisados (los alumnos), y la evaluación de su desempeño.

Esta visión informal parece más alineada a la tercera ola de SILVA, GIMBERT y NOLAN (2000) o a una posible "cuarta ola" propuesta por POUNDER (2006). Él considera que una de las expectativas básicas de un profesor líder es que sea un ejemplo de enseñanza en el aula y, de esta manera, un camino válido para la investigación del liderazgo de los profesores sería examinar las características que un profesor presenta en el aula.

TRESLAN (2006) parece en acuerdo con eso ya que considera que la jerarquía no es importante en el contexto de las clases. Para él, un líder es un individuo que actúa en un grupo con intereses, propósitos y objetivos comunes y que influye sobre los esfuerzos de este grupo para el logro de sus objetivos. POUNDER continúa estudiando esa visión (2014), o sea, que un profesor es un líder e interactúa con los alumnos para facilitarles el alcance de sus objetivos individuales y los del grupo.

Una posible explicación de la relación entre las características de los profesores líderes y su excelente performance en la clase es que estos muestran características de liderazgo transformacional y eso da lugar a un excelente desempeño en el aula. Por lo tanto, el examen de los 
comportamientos en el aula de estos profesores considerados excelentes, utilizando el liderazgo transformacional como marco de referencia, podría, de alguna manera, explicar por qué los profesores excelentes tienden a convertirse en profesores líderes y, por el contrario, por qué los profesores líderes son generalmente excelentes en el aula. Es posible que estas personas posean cualidades de liderazgo transformacional que las haga eficaces tanto en las áreas organizativas de la escuela como en las clases (POUNDER, 2006).

TRESLAN (2006) afirma que, aplicado a la clase, el liderazgo transformacional pone en foco la relación entre los profesores eficaces y sus estudiantes. Estos profesores se preocupan por que sus estudiantes vayan más allá de sus propios intereses y al bien de toda la escuela. Aquí las transformaciones incluirían elevar los niveles de conciencia de los alumnos, ayudándoles a buscar la realización personal, la comprensión de la necesidad de cambio y la construcción de la confianza.

TRESLAN (2006) entiende que el liderazgo transformacional tiene como punto de partida la creencia de que la gente quiere sentirse empoderada. Para él, los profesores están en una privilegiada posición para testimoniar el liderazgo como un proceso de aprendizaje recíproco y que implica la toma compartida de decisiones, la colaboración, la confianza y el empoderamiento. Así, refuerza la conceptualización no tradicional, no jerárquica del liderazgo, en el sentido que los líderes pasan a ser vistos como gerentes del significado, exhibiendo comportamientos inspiradores, visionarios y simbólicos.

Entonces, TRESLAN (2006) concluye que el liderazgo docente en clase (aunque subestimado) existe e impacta significativamente sobre la eficacia escolar. Que la poca atención que se presta a la contribución del liderazgo de los profesores en el cumplimiento de sus responsabilidades profesionales como administradores del proceso de aprendizaje puede ser debida, en parte, a una persistente expectativa burocrática (relacionada con la jerarquía) o a la creencia tradicional de que el liderazgo docente es, en el mejor de los casos, periférico a la eficacia de la enseñanza. Pero lo que concluye es que, dado que muchas escuelas descuidan el liderazgo de profesores, pueden ser llevados a cabo esfuerzos para ayudar a los profesores en la consecución de la práctica docente efectiva con fines de proporcionar beneficios asociados con el liderazgo transformacional, como el aprendizaje cognitivo y afectivo.

MUZIS (2008) encontró en la literatura que profesores que se preocupan con la enseñanza de calidad reconocen que la gestión del aula es un aspecto importante (con lo que HARRISON, 2011, y TRESLAN, 2006, están en acuerdo) y que ella es una variable influyente en la efectividad de los profesores, ya que los profesores/ gestores eficaces son capaces de aumentar el involucramiento de los estudiantes en el aprendizaje, aprovechar cada momento de instrucción y, entre otras cosas:

- muestran una actitud positiva que contagia a todos;

- establecen rutinas para todas las tareas y necesidades diarias;

- descubren un equilibrio entre la variedad y desafío en las actividades para los estudiantes;

- orquestan transiciones suaves y la continuidad del impulso a lo largo del día, respondiendo al flujo y reflujo de los estudiantes;

- usan el espacio y la proximidad o el movimiento por la clase para estar cerca de focos de conflicto y fomentar la atención;

- establecen normas y procedimientos, los enseñan, los ensayan en el contexto en el que se aplicarán y hacen con que todos los cumplan; 
- resuelven problemas menores de falta de atención y disrupciones, antes de que se conviertan en grandes trastornos.

Según TRESLAN (2006), y en acuerdo con la teoría del liderazgo transformacional, los profesores que presentan este liderazgo son carismáticos; capaces de crear una visión clara para la clase; dedicados a fomentar el desarrollo personal; adeptos de la práctica de empoderamiento; innovadores; predican con el ejemplo; involucran a los estudiantes a hacer del aprendizaje una experiencia divertida, para que ellos, a su vez, estén motivados por una creencia de que lo que el profesor está transmitiendo es fundamental para su educación.

De hecho, para demonstrar las similitudes entre la enseñanza efectiva y el liderazgo transformacional, TRESLAN (2006) hace dos comparaciones: una entre los componentes de los valores del liderazgo transformacional con los de los dominios de la enseñanza efectiva y otra entre las actividades del aula relacionadas con los valores del liderazgo transformacional y los dominios de la enseñanza efectiva (él las organizó en dos tablas expuestas en su artículo).

El liderazgo transformacional puede ser una posible respuesta al desafío de mejorar el pensamiento crítico de los alumnos, identificado por CRUZ, PAYAN-CARREIRA, DOMINGUEZ (2017), ya que BOLKAN, GOODBOY y GRIFFIN (2011) descubrieron que cuando los profesores influencian la motivación intrínseca de los alumnos a través del uso de comportamientos intelectualmente estimulantes, los alumnos abordan su aprendizaje de forma profunda (buscan significado en la materia y la relacionan con otras experiencias e ideas, con un enfoque crítico) y estratégica (estudian de manera organizada y esforzada, y optimizan el éxito a través del uso efectivo del espacio y el tiempo).

\section{EL LIDERAZGO DE LOS PROFESORES Y LA SATISFACCIÓN DEL CLIENTE/ALUMNO}

La influencia positiva del liderazgo transformacional sobre el esfuerzo y la satisfacción de los subordinados, la cooperación y cumplimiento, y el desempeño ha sido ampliamente estudiada (BASS, 1985, 1990, 1999). POUNDER (2014) reunió varios estudios en ese sentido y también encontró evidencias que indican mejoras en el aprendizaje de subordinados. Él, entonces, defiende (2006) que sería de gran relevancia para la educación investigar si los beneficios encontrados en las investigaciones acerca del liderazgo transformacional en contextos organizacionales, tales como la mejora del aprendizaje, la innovación, la creatividad y el comportamiento ético, también sean observados en el aula.

El propio POUNDER (2004) ya había producido evidencias en ese sentido al conducir un análisis de la aplicabilidad del liderazgo transformacional en clases en una escuela de negocios de la Universidad de Hong Kong, con una muestra de 475 estudiantes universitarios. Su investigación indicó que los profesores percibidos como líderes transformacionales en clases influyeron positivamente en:

- El esfuerzo extra auto-reportado por parte de los estudiantes;

- Su percepción de eficacia del profesor;

- La satisfacción con sus profesores.

HARRISON (2011) encontró en la literatura diversos efectos positivos del liderazgo transformacional de profesores en la instrucción, demostrados en:

- Niveles más altos de participación de los estudiantes debido a los comportamientos de motivación inspiradora, consideración individualizada y estimulación intelectual por parte del profesor; 
- Niveles más altos de respeto, satisfacción y confianza de los estudiantes en el profesor que tenga carisma (o influencia idealizada) y les estimule intelectualmente (estimulación intelectual);

- Mejor rendimiento de los estudiantes, niveles más altos de aprendizaje afectivo, motivación, gestión del conocimiento, de confianza en el profesor que motiva, entrena (hace coaching), estimula intelectualmente y exhibe carisma;

- La disposición del estudiante para ejercer esfuerzos extras, niveles más altos de percepción de eficacia y de satisfacción general con el profesor, por medio del carisma, de la consideración individualizada, estimulación intelectual y motivación inspiradora;

- Mejores resultados de los estudiantes en términos de aprendizaje afectivo, percepción de credibilidad del profesor y motivación, debido al carisma y la consideración individualizada;

- Niveles más altos de aprendizaje cognitivo y afectivo, motivación y satisfacción con la comunicación del profesor, debido al carisma del instructor, estimulación intelectual y motivación inspiradora.

Para alcanzar los objetivos de cómo mejorar el clima, el proceso educativo, la experiencia educativa de los alumnos (así como su propia excelencia en clase) y participar en la solución de problemas y en el rediseño de las escuelas, POUNDER (2006) identificó características de liderazgo que los profesores líderes eficaces de la tercera ola manifiestan y las asoció con conceptos de liderazgo transformacional (Carisma o Influencia idealizada, que puede incluir atributos, comportamientos y motivación inspiradora, Consideración individual y Estimulación intelectual):

- Influencia idealizada y motivación inspiradora: demuestran un profundo compromiso con un conjunto de valores fundamentales; los comunican abiertamente; exhiben un entusiasmo contagioso, una capacidad de inspirar a los demás y de aumentar sus expectativas;

- Consideración individual: desarrollan confianza y relaciones (incluso fomentando la participación de colegas), son modelos de crecimiento profesional, hacen mentoring, coaching y modelan el aprendizaje;

- Estimulación intelectual: fomentan el cambio y desafían la situación actual, el status quo, en especial para revisar y mejorar la práctica escolar, estimulando nuevas formas de hacer las cosas, buscan su propio desarrollo profesional y el de sus colegas, lo que incluye investigaciones académicas, grupos de estudio y la observación de formas efectivas de enseñanza para reproducirlas.

HARRISON (2011), estudiando el contexto online, recolectó datos empíricos que mostraron una correlación positiva significativa, en acuerdo con la literatura que examina estos mismos conceptos en las aulas tradicionales, entre los comportamientos de liderazgo transformacional del instructor y los resultados de los estudiantes, tales como Aprendizaje cognitivo (comprender la información, organizar las ideas, analizar y sintetizar los datos, aplicar los conocimientos, la elección entre alternativas en la resolución de problemas y evaluar las ideas o acciones); Aprendizaje afectivo (sentimientos positivos hacia la materia, instructor y curso); Credibilidad del profesor (percepciones de los estudiantes acerca de la competencia del profesor, de su honradez y buena voluntad); La satisfacción con la comunicación (respuesta afectiva del estudiante acerca de la realización de los objetivos y expectativas de comunicación): asociada con el componente influencia idealizada del liderazgo transformacional y que consiste en comunicar una visión, utilizando un lenguaje motivacional, y articular los medios para llevar a cabo la visión. 
Analizando resultados de estudiantes turcos en un examen internacional estandarizado de matemáticas, YAVUZ, YALÇIN y DIBEK (2017) encontraron que su rendimiento en matemáticas tiene relación negativa significativa con estar sujetos a intimidación (bullying) en la escuela y una relación positiva significativa con el énfasis del profesor en el logro académico y con los estudiantes que les gusta aprender las matemáticas, dos condiciones que pueden ser asociados con el carisma o la influencia idealizada del liderazgo transformacional.

JACQUES, GARGER y VRACHEVA (2016) identificaron que, en los Estados Unidos, un número creciente de IESs ha introducido el aprendizaje de servicios (service-learning) como una estrategia pedagógica que integra el servicio comunitario al estudio académico para enriquecer el aprendizaje, enseñar responsabilidad cívica e incentivar la participación de estudiantes para fortalecer sus comunidades. En este contexto, en general, hay dos líderes: el instructor del curso y un interlocutor de la comunidad o cliente, que recibe el producto de los esfuerzos de los alumnos involucrados en el proyecto. Incluso con estos dos líderes, los resultados mostraron que las percepciones de los estudiantes sobre el liderazgo transformacional de los instructores estaban asociadas con la relevancia del curso, la intención de involucrarse con los asuntos de la comunidad y la satisfacción con el aprendizaje de servicios.

TSAI y LIN (2012) investigaron si el modelo de liderazgo transformacional podría ser adaptado para evaluar a los profesores de manera más próxima de la realidad de las clases que los cuestionarios comúnmente utilizados actualmente. Su investigación (TSAI y LIN, 2012), centrada en estudiantes universitarios, encontró evidencias en la literatura de que los comportamientos de liderazgo transformacional en el aula poseen una correlación positiva con el compromiso o participación (previamente asociados con aprendizaje) que se basa en el que BASS (1985), el autor que más desarrolló el concepto del liderazgo transformacional, originalmente identificado por BURNS (1978), ha llamado "esfuerzo extra", y la satisfacción de los estudiantes. Sus datos empíricos (TSAI y LIN, 2012) revelaron una significativa correlación moderadamente positiva con el compromiso y la satisfacción de los estudiantes. El nivel de interés de los estudiantes del curso también mostró una significativa relación moderadamente positiva con su compromiso y satisfacción.

BALWANT (2016) realizó un meta-análisis con 22 estudios empíricos que corroboró los beneficios del liderazgo transformacional de profesores/ instructores en el aula, hallando correlación positiva con la motivación, la satisfacción, las percepciones de la credibilidad del instructor, el rendimiento académico, el aprendizaje afectivo y el aprendizaje cognitivo de los estudiantes.

\section{RESULTADOS}

La Tabla 2, presentada a continuación, exhibe los autores de la literatura que hallaron evidencias de la influencia positiva del liderazgo transformacional (solo o en combinación con el liderazgo transaccional) sobre la satisfacción y el aprendizaje (un estudio identificado con "1" es un meta-análisis). 


\begin{tabular}{|l|l|}
\hline $\begin{array}{c}\text { Satisfacción y Aprendizaje generados } \\
\text { por el liderazgo transformacional }\end{array}$ & \multicolumn{1}{|c|}{ Autores/ Trabajos } \\
\hline Satisfacción & JACQUES, GARGER y VRACHEVA, 2016; \\
& BALLWANT, 2016; \\
& TSAI y LIN, 2012; \\
& HARRISON, 2011; \\
& LIVINGSTON, 2011; \\
& BOLKAN y GOODBOY, 2009; \\
& GOODBOY y MYERS, 2008; \\
& POUNDER, 2004; \\
& WALUMBWA, WU y OJODE, 2004; \\
& HARVEY, ROYAL y STOUT, 2003; \\
& WALUMBWA y OJODE, 2000. \\
\hline Aprendizaje & BALLWANT, 20161; \\
& DANIELS y GOODBOY, 2014; \\
& HARRISON, 2011; \\
& BOLKAN, GOODBOY y GRIFFIN, 2011; \\
& BOLKAN y GOODBOY, 2010; \\
& BOLKAN y GOODBOY, 2009; \\
& GOODBOY y MYERS, 2008; \\
& POGUE y AHYUN, 2006. \\
\hline
\end{tabular}

Tabla 2: Evidencias empíricas de satisfacción y el aprendizaje asociados con el liderazgo transformacional (solo o en combinación con el liderazgo transaccional)

Fuente: elaboración propia

Es importante resaltar aún que ciertos autores, como CHAMBEL y CURRAL (2005) y BEATTY y ZHAN (1990), encontraron evidencia de que la satisfacción, uno de los resultados comúnmente asociados con el liderazgo transformacional, puede, por sí solo, producir efectos beneficiosos en el aprendizaje.

La Tabla 3, a continuación, presenta los autores de la literatura relevante que encontraron evidencia empírica de otros resultados que son comúnmente asociados con el liderazgo transformacional (solo o en combinación con el liderazgo transaccional) y que pueden ser útiles en el contexto de clase de aulas (un estudio identificado con "1" es un meta-análisis).

\begin{tabular}{|l|l|}
\hline $\begin{array}{l}\text { Otros logros generados por el } \\
\text { liderazgo transformacional }\end{array}$ & \multicolumn{1}{|c|}{ Autores/ Trabajos } \\
\hline Esfuerzo Extra & $\begin{array}{l}\text { LIVINGSTON, 2011; BOLKAN y GOODBOY, 2009; POUNDER, 2004; } \\
\text { WALUMBWA, WU y OJODE, 2004; WALUMBWA y OJODE, 2000; }\end{array}$ \\
\hline Motivación & $\begin{array}{l}\text { BALLWANT, 2016'; WILSON et al., 20121; BOLKAN, GOODBOY y } \\
\text { GRIFFIN, 2011; BOLKAN y GOODBOY, 2009; GOODBOY y MYERS, } \\
\text { 2008; POGUE y AH YUN, 2006; }\end{array}$ \\
\hline Participación superior en el aula & $\begin{array}{l}\text { TSAI y LIN, 2012; WILSON et al., 20121; BOLKAN y GOODBOY, 2009; } \\
\text { GOODBOY y MYERS, 2008; HARVEY, ROYAL y STOUT, 2003; }\end{array}$ \\
\hline $\begin{array}{l}\text { Percepción de eficacia del líder } \\
\text { (profesor) en el aula }\end{array}$ & $\begin{array}{l}\text { LIVINGSTON, 2011; POUNDER, 2004; WALUMBWA, WU y OJODE, } \\
\text { 2004; HARVEY, ROYAL y STOUT, 2003; }\end{array}$ \\
\hline $\begin{array}{l}\text { Percepción de credibilidad del líder } \\
\text { (profesor) }\end{array}$ & $\begin{array}{l}\text { BALLWANT, 20161; HARRISON, 2011; BOLKAN y GOODBOY, 2009; } \\
\text { POUNDER, 2004; HARVEY, ROYAL y STOUT, 2003; WALUMBWA y } \\
\text { OJODE, 2000; }\end{array}$ \\
\hline
\end{tabular}

Tabla 3: Evidencias empíricas de logros asociados con el liderazgo transformacional (solo o en combinación con el liderazgo transaccional).

Fuente: elaboración propia 


\section{CONCLUSIÓN}

La investigación bibliográfica acerca de las contribuciones del liderazgo instruccional que conduzcan a una mayor satisfacción de los alumnos apuntó que el liderazgo de profesores puede ser útil a las instituciones de enseñanza en sus esfuerzos para destacarse y aumentar su competitividad en un mercado cada vez más disputado.

En cuanto a la cuestión de investigación:

\section{¿Existen evidencias de que el liderazgo educativo puede generar beneficios para la satisfacción de los alumnos?}

$\mathrm{Si}$, existen suficientes evidencias de que el liderazgo con fines instruccionales, en particular el liderazgo transformacional practicado por los profesores, puede generar beneficios significativos para la satisfacción de los alumnos, como se puede ver en la Tabla 2.

Fueron encontradas aún evidencias de que el liderazgo de profesores puede generar beneficios para el aprendizaje de los alumnos, ya que la satisfacción con el profesor puede mejorar el aprendizaje como apuntan CHAMBEL y CURRAL (2005) y BEATTY y ZHAN (1990). Otros investigadores relacionaron más uno de los resultados del liderazgo transformacional, el esfuerzo extra, con mejoras en el aprendizaje, como RICHARDSON, ABRAHAM y BOND (2012); BOLKAN y GOODBOY (2009); y CARBONARO (2005). También fueron encontradas evidencias de que el liderazgo instruccional compartido entre los directores y profesores resultó en logros pedagógicos y estudiantiles.

Como sugestiones para investigaciones futuras, se recomienda que se realicen más estudios empíricos con muestras aleatorias y significativas para corroborar los efectos positivos del estilo transformacional de liderazgo en la satisfacción de los alumnos, tanto en la escuela como en la educación superior, en especial en las escuelas de negocios (business schools), en todos los continentes. Además, si aumentos en la satisfacción pueden tener relación con la empleabilidad de los exalumnos, por ejemplo.

Como se encontró evidencia de los impactos de la satisfacción y del esfuerzo extra de los alumnos sobre el aprendizaje, surge la oportunidad de investigarse si otros efectos indirectos del liderazgo transformacional, como la credibilidad de profesores y la percepción de eficacia del profesor, también pueden hacerlo. Además, si pueden producir otros beneficios relevantes.

Este artigo encontró, presentó y organizó evidencias que muestran que el liderazgo con objetivos instruccionales, en especial el liderazgo transformacional de profesores, presenta oportunidades para mejorar la satisfacción de los alumnos y, por ello, probablemente también la competitividad de las instituciones de enseñanza superior.

\section{REFERENCIAS}

ARSLANAGIĆ-KALAJDŽIĆ, Maja; KADIĆ-MAGLAJLIĆ, Selma; ČIČIĆ, Muris. Students' Perceptions about Role of Faculty and Administrative Staff. Business Education Service Quality Assessment. Tržište, v. 26, n. 1, p. 93-108, 2014.

BALWANT, Paul T. Transformational Instructor-Leadership in Higher Education Teaching: A Meta-Analytic Review and Research Agenda. Journal of Leadership Studies, v. 9, n. 4, Winter, pp. 20-42, 2016. DOI: 10.1002/jls.21423 BASS, Bernard M. Leadership and performance beyond expectations. Free Press; Collier Macmillan, 1985.

BASS, Bernard M. Two decades of research and development in transformational leadership. European journal of work and organizational psychology, v. 8, n. 1, p. 9-32, 1999.

BASS, Bernard M. From transactional to transformational leadership: learning to share the vision, Organizational Dynamics, v. 18, n. 3, p. 19-36, 1990. 
BEATTY, Michael J.; ZAHN, Christopher J. Are student ratings of communication instructors due to "easy" grading practices?: An analysis of teacher credibility and student-reported performance levels. Communication Education, v. 39, n. 4, p. 275-282, 1990.

BOLKAN, San; GOODBOY, Alan K.; GRIFFIN, Darrin J. Teacher Leadership and Intellectual Stimulation: Improving Students' Approaches to Studying through Intrinsic Motivation. Communication Research Reports, v. 28, n. 4, p. 337-346, 2011.

BOLKAN, San; GOODBOY, Alan K. Transformational leadership in the classroom: The development and validation of the student intellectual stimulation scale. Communication Reports, v. 23, n. 2, p. 91-105, 2010.

BOLKAN, San; GOODBOY, Alan K. Transformational leadership in the classroom: Fostering student learning, student participation, and teacher credibility. Journal of Instructional Psychology, v. 36, n. 4, p. $296,2009$.

BRISCO, G. F. M.; GILlI, J. J.; ARMENGOL, M. L.; COLOMBO, M. P. Higher Education Quality: What is the perception of the players involved? conclusions of a comparative analysis. Visión de Futuro, v. 20, n. 1, p. 24-43, 2015.

BURNS, J. M. Leadership. New York: Harper \& Row, 1978.

CARBONARO, William. Tracking, students' effort, and academic achievement. Sociology of Education, v. 78, n. 1, p. 27-49, 2005

CHAMBEL, Maria José; CURRAL, Luís. Stress in academic life: work characteristics as predictors of student well-being and performance. Applied psychology, v. 54, n. 1, p. 135-147, 2005.

CRUZ, G., PAYAN-CARREIRA, R., \& DOMINGUEZ, C. Critical thinking education in the Portuguese higher education institutions: A systematic review of educational practices. Revista Lusófona de Educação, v. 38, n. 38, pp. 43-61, 2017. Disponível em http://recil.ulusofona.pt/bitstream/handle/10437/8604/Critical\%20thinking\%20 education.pdf?sequence $=1$, acesso em 22/06/2018.

DANIELS, Rita; GOODBOY, Alan K. Transformational leadership in the Ghanaian university classroom. Intercultural Communication Studies, v. 23, n. 2, 2014.

DLAČIĆ, Jasmina; ARSLANAGIĆ, Maja; KADIĆ-MAGLAJLIĆ, Selma; MARKOVIĆ, Suzana; RASPOR, Sanja. Exploring perceived service quality, perceived value, and repurchase intention in higher education using structural equation modelling. Total Quality Management \& Business Excellence, v. 25, n. 1-2, pp. 141-157, 2014.

FROEMMING, Lurdes M. S. Encontros de serviços em uma instituição de ensino superior. 2001. Tese de Doutorado-Escola de Administração, Universidade Federal do Rio Grande do Sul, Porto Alegre, RS, 2001.

GOODBOY, Alan K.; MYERS, Scott A. The effect of teacher confirmation on student communication and learning outcomes. Communication Education, v. 57, n. 2, p. 153-179, 2008.

HARRIS, Alma. Teacher leadership as distributed leadership: heresy, fantasy or possibility? School leadership \& management, v. 23, n. 3, p. 313-324, 2003.

HARRISON, Janelle L. Instructor transformational leadership and student outcomes. Emerging leadership journeys, v. 4, n. 1, p. 91-119, 2011.

HARVEY, Steve; ROYAL, Martin; STOUT, Dale. Instructor's transformational leadership: University student attitudes and ratings. Psychological reports, v. 92, n. 2, p. 395-402, 2003.

HILL, Yvonne; LOMAS, Laurie; MACGREGOR, Janet. Students' perceptions of quality in higher education. Quality assurance in education, v. 11, n. 1, p. 15-20, 2003.

JACQUES, Paul H.; GARGER, John; VRACHEVA, Veselina. The Effects of Two-Source Transformational Leadership on Student Outcomes of Service-Learning Projects. Journal of Behavioral and Applied Management, v. 17, n. 2, p. 152, 2016.

LEBLANC, Gaston; NGUYEN, Nha. Searching for excellence in business education: an exploratory study of customer impressions of service quality. International Journal of Educational Management, v. 11, n. 2, p. 72-79, 1997.

LEITHWOOD, Kenneth; SUN, Jingping. The nature and effects of transformational school leadership: a meta-analytic review of unpublished research. Educational Administration Quarterly, v. 48, n. 3, p. 387-423, 2012.

LEITHWOOD, Kenneth; SEASHORE LOUIS, K.; ANDERSON, S.; WAHLSTROM, K. Review of research: How leadership influences student learning. The Wallace Foundation: 2004.

LEITHWOOD, Kenneth A.; RIEHL, Carolyn. What we know about successful school leadership. Laboratory for Student Success, Temple University, Philadelphia, PA, 2003.

LIVINGSTON, Robert K. An investigation of transformational leadership in a virtual learning environment. 2010. Tese de Doutorado. Capella University, Minneapolis, MN, 2011.

MARKS, H. M. PRINTY, S. M. Principal leadership and school performance: Integration of transformational and instructional leadership. Educational Administration Quarterly, 39(3), p. 370-397, 2003.

MELCHOR CARDONA, Madeline; BRAVO, Juan José. Service quality perceptions in higher education institutions: the case of a Colombian university. Estudios Gerenciales, v. 28, n. 125, p. 23-29, 2012.

MUZIS, Ivars. Learning-Centered Leadership. In: ATEE Spring University Conference "Teacher of the 21st Century: Quality Education for Quality Teaching”, 2008, Riga. Proceedings... Riga: University of Latvia. p. 605-610, 2008. 
NEUMERSKI, Christine M. Rethinking Instructional Leadership, a Review: What Do We Know About Principal, Teacher, and Coach Instructional Leadership, and Where Should We Go From Here? Educational administration quarterly, v. 49, n. 2, p. 310-347, 2012.

PELLICIARI, Marcos Roberto de Mendonça. A liderança do docente como elo integrador no processo ensinoaprendizagem baseado em problemas (Problem-Based Learning) em ambientes híbridos na engenharia. 2013. Dissertação (Mestrado em Economia, organizações e gestão do conhecimento)-Escola de Engenharia, Universidade de São Paulo, São Carlos, SP, 2013.

POGUE, Lanette L.; AHYUN, Kimo. The effect of teacher nonverbal immediacy and credibility on student motivation and affective learning. Communication Education, v. 55, n. 3, p. 331-344, 2006.

POUNDER, James S. The classroom leadership styles of Hong Kong University teachers: a case study of teachers in a business school. 2004. Tese de Doutorado-Educação, University of Leicester, United Kingdom, 2004.

POUNDER, James S. Full range leadership in the university classroom: a Hong Kong study. Hong Kong: Institute of Business Studies, Paper 41, 2005 (Working Paper Series). Disponível em: 〈 http://commons.ln.edu.hk/hkibswp/41〉. Acesso em: 28/01/2016.

POUNDER, James S. Transformational Classroom Leadership: The Fourth Wave of Teacher Leadership? Educational Management Administration \& Leadership, v. 34, n. 4, p. 533-545, 2006.

POUNDER, James S. Quality teaching through transformational classroom leadership. Quality Assurance in Education, v. 22, n. 3, p. 273-285, 2014.

RICHARDSON, Michelle; ABRAHAM, Charles; BOND, Rod. Psychological correlates of university students' academic performance: a systematic review and meta-analysis. Psychological bulletin, v. 138, n. 2, p. $353,2012$.

ROBBINS, Steven B.; LAUVER, Kristy; LE, Huy; DAVIS, Daniel; LANGLEY, Ronelle; CARLSTROM, Aaron. Do psychosocial and study skill factors predict college outcomes?: A meta-analysis. Psychological Bulletin, v. 130, n. 2, p. 261-288, 2004.

SCHWANKE, Rúbia B. Processo de avaliação da satisfação do cliente em instituições de ensino superior: um estudo nas Comissões Próprias de Avaliação. 2013. Dissertação (Mestrado em Desenvolvimento). Universidade Regional do Noroeste do Estado do Rio Grande do Sul - UNIJUÍ, RS, 2014.

SILVA, Diane; GIMBERT, Belinda; NOLAN, James. Sliding the doors: Locking and unlocking possibilities for teacher leadership. The Teachers College Record, v. 102, n. 4, p. 779-804, 2000.

TSAI, Kuan Chen; LIN, Kate. Rethink student evaluation of teaching. World Journal of Education, v. 2, n. 2, p. p17, 2012.

TRESLAN, Dennis L. Transformational Leadership in the Classroom: Any Evidence? Education Canada, v. 46, n. 2, p. 58-62, 2006.

WALUMBWA, O.; OJODE, L. A. Gender stereotype and instructors' leadership behavior: Transformational and transactional leadership. In: Midwest Academy of Management Annual Conference, March 30th-April $1^{\text {st }}$, 2000 , Chicago. Proceedings... Chicago, IL: Midwest Academy of Management, 2000. Disponível em: https://www.researchgate.net/profile/Lucy_Ojode/publication/228551285_Gender_stereotype_and instructors' leadersh ip behavior_Transformational_and transactional_leadership/links/0deec53b6eb135e618000000.pdf. Acesso em: 19/02/2016.

WALUMBWA, O.; WU, Cindy; OJODE, Lucy A. Gender and instructional outcomes: The mediating role of leadership style. Journal of Management Development, v. 23, n. 2, p. 124-140, 2004.

WILliAMS, Robert L.; CLARK, Lloyd. Academic Causal Attributions and Course Outcomes for College Students. 2002. ERIC ED 469337, ERIC Project, Washington, DC.

WILSON, A. J.; LIU, Y.; KEITH, S. E.; WILSON, A. H.; KERMER, L. E.; ZUMBO, B. D.; BEAUCHAMP, M. R. Transformational teaching and child psychological needs satisfaction, motivation, and engagement in elementary school physical education. Sport, Exercise, and Performance Psychology, v. 1, n. 4, p. 215, 2012.

YAVUZ, Hatice Ç.; YALÇIN, S.; DIBEK, Münevver I. The effects of student and teacher level variables on TIMSS 2007 and 2011 mathematics achievement of Turkish students. Egitim ve Bilim (Education and Science), v. 42, n. 189, 2017.

YORK-BARR, Jennifer; DUKE, Karen. What do we know about teacher leadership?: Findings from two decades of scholarship. Review of educational research, v. 74, n. 3, p. 255-316, 2004.

This work is licensed under a Creative Commons Attribution 4.0 International License. 